\title{
CASE OF PRIMARY LATERAL SCLEROSIS OR SPASMODIC TABES.
}

BY JOHN HOPKINS, F.R.C.S., ENG.

Medical Superintendent, Cleveland Street Sick A8ylum, W.

J. M., aged 21, porter, was admitted August 14th, 1881 . Father and mother living, and stated to be in good health. All brothers and sisters, thirteen in number, died under four years of age, two of them of smallpox.

Patient had fits when a child. Never had venereal disease, smallpox, or any illness till twelve months ago, when he got wet, and within a week found his legs becoming weak and dragging, and his gait tottering; he improved a little, but the weakness and tottering never left him entirely.

The following was the condition on admission. Patient is fat but pasty-looking, and feels in perfect health. No signs of congenital syphilis. Gait peculiar, rolls from side to side, and as each limb is brought forward it jerks irregularly, and when put down stiffens and trembles. No difficulty in standing with eyes closed. On tip-toe the legs tremble. Percussion of the patellar tendon causes not unly heightened patellar reflex, but trembling of the limb. Ankle clonus marked. Slight loss of sensation in the sole of the right foot (?) No wasting of muscles. Arms not affected. No symptoms referable to special senses, tongue, stomach or bladder. Fundus oculi normal. Heart and lungs free from signs of organic lesion. Urine clear and free from albumen.

Patient for some months got up daily, ate and slept well, and expressed himself as feeling perfectly well, but walking became increasingly difficult, and all the symptoms aggravated. Passive elevation of the knee from the bed, or attempt at producing ankle clonus threw, first the one, then both limbs into violent trembling. For a time crutches were used, but had to be abandoned. On February 1st, 1882, it was noticed by the patient when he tried to stand, that there was scarcely any feeling in the soles of the feet; the ground, which simply felt warm to the feet, seemed to be sinking under them. On test- 
ing them, the prick of a pin was readily felt. Rigid flexion was noticed to come on for the first time on May 16th. It first occurred in the right lower limb, but in a few days affected the left also. The limbs could not then be extended passively. The spasm was not permanent; at times they could be extended voluntarily. On May 19 th the urine was alkaline and purulent, and bed sores had formed over the sacrum and trochanters. A night's rest in a sitting posture gave a gangrenous look to the whole nates. However they recovered again. Wasting of the muscles at the front and back of the legs was noted then for the first time. On June 15th, patient complained of stiffness of the upper limbs. On examination they yielded very slowly to passive movement, both in flexion and extension, but there was no spasm that could not be overcome. There was no pain, tenderness, heightened reflex, or clonus in them.

The bed sores penetrated deeply to the bones through thick pads of fat. During the last week the temperature steadily rose, with remissions, accompanied by rigors and sweats, to $105-8^{\circ} \mathrm{F}$. This was on July 12 th : on the same day, the bed sore over the right trochanter bled freely. The next day the patient died. Temperature normal an hour before death.

Autopsy.-Body fat; lower limbs wasted. Serous cavities, heart, lungs, intestinal tract, liver spleen and kidneys normal. Rugæ of bladder congested, and trigone covered with mucus.

Brain and membranes, spinal canal and dura mater healthy.

The spinal cord presented marked gelatinous degeneration in the lateral columns which extended from the tip upwards into the upper portion of the dorsal region. The degeneration extended higher in the right side, and at a point above any visible sclerosis the column was congested, being in marked contrast to the rest of the white matter. In the lumbar enlargement the degeneration extended forwards into the anterior column and in front of this the white matter was greyer and firmer than normal. In one spot the anterior columns next the anterior median fissure were gelatinous. $A$ bove the lumbar enlargement the sclerosis did not invade the anterior columns to the same extent, and higher still the lateral columns were only in part affected. In the upper part of the dorsal region the gelatinous degeneration was visible as a thin band, just in front of the posterior cornu and parallel with it. After hardening in chromic acid the parts described above as sclerosed were stained brown, the tint varying with the degree of degeneration. Besides, the central grey matter was also stained in a similar manner in certain parts. In the mid-dorsal region and below this (except in the largest part of the lumbar enlargement), the grey matter was throughout darker than at the upper part of the cord. Above the mid- 
dorsal region the brown staining invaded less and less of the grey matter, the anterior cornua being free from it, while the posterior half of the grey mass was stained. Higher still, at a point where the sclerosis in the lateral columns was shown by a thin wedge-shaped tract on the right side, and a thin band on the left, the grey matter was very little discoloured.

Microscopic examination showed the lateral columns and neighbouring part of the anterior to be nearly devoid of nerve tubes at the lower end of the cord, and the anterior columns to contain a considerable quantity of connective tissue between the nerve tubes. In the dorsal region the lateral columns contained some nerve tubes, which were found in increasing numbers as the examination was extended upwards. The most sclerosed part of the column seemed to be not directly in contact with the posterior cornu. In the middorsal region the anterior columns were comparatively free from connective tissue. The large cells of the anterior cornua were much atrophied in the lower half of the cord, except in one section taken from the lumbar enlargement; in this the cells appeared normal in size, and number; but a little lower where the cells were in three pretty well defined groups, a small central group, a larger antero,-lateral and a large posterolateral group, the two first groups were markedly atrophied on the right side.

Remarks.-In 'The Localization of Cerebral and Spinal Diseases," Charcot, in attributing the symptoms of spasmodic tabes to primary sclerosis of the lateral columns, says "the hypothesis at the present time has received no verification." 2 Although in the above case the sclerosis extended beyond the lateral columns at the lower end of the cord, the symptoms, taken together with the extent and degree of sclerosis of these columns found post mortem, make it more than probable that this was a case of primary ascending sclerosis of the lateral columns.

1 New Sydenham Socicty's Translation, p. 304.

2 Dr. Dreschfeld has published a caso since Charcot published this: vide British Mod. Journ. 1881. 